\title{
Positron Range Effects in High Resolution 3D PET Imaging
}

\author{
J. Cal-González, J. L. Herraiz, S. España, M. Desco, J. J. Vaquero, J. M. Udías
}

\begin{abstract}
Positron range limits the spatial resolution of PET images. It has a different effect for different isotopes and propagation materials, therefore it is important to consider it during image reconstruction, in order to obtain the best image quality. Positron range distribution was computed using Monte Carlo simulations with PeneloPET. The simulation models positron trajectories and computes the spatial distribution of the annihilation coordinates for the most common isotopes used in PET: ${ }^{18} \mathrm{~F},{ }^{11} \mathrm{C},{ }^{13} \mathrm{~N},{ }^{15} \mathrm{O},{ }^{68} \mathrm{Ga}$ and ${ }^{82} \mathrm{Rb}$. Range profiles are computed for different positron propagation materials, obtaining one kernel profile for each isotope-material combination. These range kernels were introduced in FIRST, a 3D-OSEM image reconstruction software, and employed to blur the object during forward projection. The blurring introduced takes into account the material in which the positron is annihilated, obtained for instance from a CT image. In this way, different positron range corrections for each material in the phantom are considered. We compare resolution and noise properties of the images reconstructed with and without positron range modelling. For this purpose, acquisitions of an Image Quality phantom filled with different isotopes have been simulated for the ARGUS small animal PET scanner.
\end{abstract}

\section{INTRODUCTION}

The range of positrons in tissue is an important limitation to the spatial resolution achievable in 3D PET [1], [2]. Recent developments in detector technology have reduced crystal size and now there are small animal PET scanners with near 1 $\mathrm{mm}$ spatial resolution, such as the ARGUS [3]. This resolution is comparable to positron range of most commonly used isotopes (see Table I). Positron range appears as a blurring of the reconstructed image. Based on measured positron range functions, Derenzo [4] proposed a method to remove the blurring in the reconstructed images in FBP. Recently, new methods to remove positron range have been developed using MAP during reconstruction [5]-[7]. In this

Manuscript received November 13, 2009. This work has been supported in part by MEC (FPA2007 62216), CDTEAM (Programa CENIT, Ministerio de Industria), UCM (Grupos UCM, 910059), CPAN (Consolider Ingenio 2010) CSPD 200700042 and the RECAVA RETIC network. Part of the calculations of this work were performed in the "Clúster de Cálculo de Alta Capacidad para Técnicas Físicas" funded in part by UCM and in part by UE under FEDER programme".

J. Cal Gonzalez, J.L. Herraiz and J M. Udías are with the Grupo de Física Nuclear, Dpto. Física Atómica, Molecular y Nuclear, UCM, Madrid, Spain (telephone: +34 91394 4484, e mail: jacobo@nuclear.fis.ucm.es)

S. España was with the Grupo de Física Nuclear, Universidad Complutense de Madrid, Spain. He is now with the Department of Radiation Oncology, Massachusetts General Hospital and Harvard Medical School, Boston, MA, USA (S.España e mail: samuel@nuclear.fis.ucm.es).

M. Desco and J.J Vaquero are with the Unidad de Medicina y Cirugía Experimental, Hospital General Universitario Gregorio Marañón, Madrid, Spain (J.J. Vaquero e mail: Juanjo@hggm.es ). work we account for positron range by modelling it effects during 3D OSEM reconstruction [8].

Positron range in water has been measured experimentally for several medically important isotopes [2], [9], [10]. These measurements show considerable variation among authors, because the resolution of the detectors was comparable to positron range. This led to the use of Monte Carlo simulations to estimate positron range [1], [11], [12]. In this work we simulate positron interactions and subsequent annihilation, with the PeneloPET code [13]. The trajectories, annihilation points, radial and x-projection profiles have been obtained.

Acquisitions of an Image Quality phantom (IQ) [14] filled with different isotopes have been simulated for the ARGUS small animal PET scanner. We compare resolution versus noise properties of the images. Preliminary results using high positron energy isotopes show significant improvement in the spatial resolution of the reconstructed images, compared to reconstructions without positron range modelling.

\section{METHODS}

\section{A. Monte Carlo Simulation}

The continuum energy spectrum distribution of emitted positrons is easily computed from theoretical grounds [1]. Positron range depends mainly on the initial energy of the positron and the number of electrons in the absorber, i.e., material density [15]. We use PeneloPET [13] for simulating positron range. PeneloPET may deal with positron range in two ways:

1. Positron trajectory and initial energy are simulated for each positron coming from the decay process. This leads to accurate results, at the expense of increasing computation time.

2. The positron annihilation point is randomly chosen from pre-computed probability distributions.

Radial and axis-projection profiles of positron range for most used isotopes and materials are included with PeneloPET. Profiles for other isotopes and other materials can be easily added with the standard tools provided with PeneloPET.

\section{$B$. Image reconstruction with positron range blurring}

Positron range correction can be introduced in iterative image reconstruction in two ways: i) using positron range profiles obtained from Monte Carlo simulations as a blurring applied to the object or ii) introducing the effect of positron range in the System Response Matrix [8]. We take the first 
method, because in this case it is possible to adapt the blurring introduced as a function of the material properties (electronic density, effective Z) of the object in which positrons are annihilated.

The positron range corrected OSEM algorithm used in this work reads:

$$
x^{\prime}{ }_{j} \equiv x_{j} \frac{\sum_{i} C_{i j}\left(\frac{y_{i}}{\sum_{j} C_{i j}, \tilde{x}_{j^{\prime}}}\right)}{\sum C_{i j}}
$$

Where $\tilde{x}_{j}$ is the object blurred by positron range which we forward project. $\widetilde{x}_{j}$, is obtained by a convolution of the initial object with a blurring function $\rho$, which can be obtained from positron range profiles [1]:

$$
\tilde{x}_{j} \equiv x_{j} \otimes \rho \equiv \frac{\sum_{h} x_{j-h} \cdot \rho_{h}}{\sum_{h} \rho_{h}}
$$

Acquisitions for the ARGUS small animal PET scanner of an Image Quality phantom [14] filled with ${ }^{18} \mathrm{~F},{ }^{11} \mathrm{C},{ }^{15} \mathrm{O}$ and ${ }^{68} \mathrm{Ga}$ isotopes have been simulated with PeneloPET. These simulated acquisitions have been reconstructed with a $3 \mathrm{D}$ OSEM procedure [8], with and without positron range modeling. To measure the image quality in our reconstructions, we compared the resolution and recovery coefficient [16] (RC) variation against noise of the image, for several iterations and subset choices.

In order to measure the effect of different materials in the positron range, we have simulated a cylinder phantom of 5 $\mathrm{cm}$ of diameter and $5 \mathrm{~cm}$ length, centered in the FOV, filled with water. It contains a $1 \mathrm{~cm}$ off-centered rod of bone material, $1 \mathrm{~cm}$ of diameter and the same length as the cylinder. Two point sources with low activity were simulated, placed at $(1,0,0) \mathrm{cm}$ inside water, and at $(-1,0,0) \mathrm{cm}$ inside bone, respectively.

\section{RESULTS}

\section{A. Monte Carlo Simulation}

Table I shows mean and maximum range of positrons in water obtained with PeneloPET, compared to theoretical values [17]. Fair agreement is obtained for all isotopes.
TABLE I: SIMULATED AND EXPERIMENTAL POSITRON RANGE IN WATER.

\begin{tabular}{ccccc} 
& $\begin{array}{c}\text { Mean range } \\
\text { PeneloPET } \\
(\mathrm{mm})\end{array}$ & $\begin{array}{c}\text { Max. range } \\
\text { PeneloPET } \\
(\mathrm{mm})\end{array}$ & $\begin{array}{c}\text { Mean range } \\
{[17](\mathrm{mm})}\end{array}$ & $\begin{array}{c}\text { Max range } \\
{[17](\mathrm{mm})}\end{array}$ \\
\hline${ }^{18} \mathrm{~F}$ & 0.61 & 2.3 & 0.64 & 2.3 \\
${ }^{11} \mathrm{C}$ & 1.04 & 3.9 & 1.03 & 3.9 \\
${ }^{13} \mathrm{~N}$ & 1.31 & 5.1 & 1.32 & 5.1 \\
${ }^{15} \mathrm{O}$ & 2.00 & 7.9 & 2.01 & 8.0 \\
${ }^{68} \mathrm{Ga}$ & 2.21 & 8.9 & 2.24 & 8.9 \\
${ }^{82} \mathrm{Rb}$ & 4.24 & 16.7 & 4.29 & 16.5
\end{tabular}

\section{B. Image reconstruction using different isotopes.}

Fig. 1 shows the comparison of the IQ filled with ${ }^{18} \mathrm{~F}$ images, obtained using 3D-OSEM, with and without positron range effects in the reconstruction. Fig. 2 shows the IQ filled with ${ }^{68} \mathrm{Ga}$ images, with and without positron range corrections in the reconstruction algorithm. In both cases, $10 \%$ noise (measured in uniform regions) images are compared.

Table II shows the resolution and $\mathrm{RC}$ values for a certain level of noise, obtained for the $3 \mathrm{~mm}$ rod of the IQ phantom, filled with ${ }^{68} \mathrm{Ga}$, reconstructed with $3 \mathrm{D}$-OSEM with and without range corrections. Images were reconstructed using 20 iterations of 10 subsets keeping the result of each iteration. We can see a significant improvement in image quality (resolution and $\mathrm{RC}$ for a given noise level) when positron range is considered during the reconstruction, especially for higher energy isotopes.

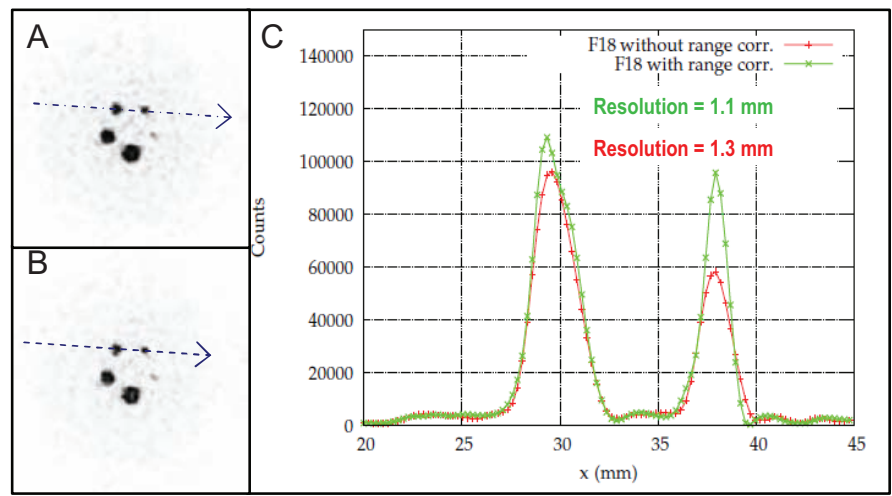

Fig. 1. IQ phantom filled with ${ }^{18} \mathrm{~F}$ reconstructed with 3D OSEM. Without range corrections (A) and with range corrections (B). In both cases, 10\% noise images are compared. In panel $\mathrm{C}$, count line profiles along the 2 and $3 \mathrm{~mm}$ rods (arrow in the images) are shown. 


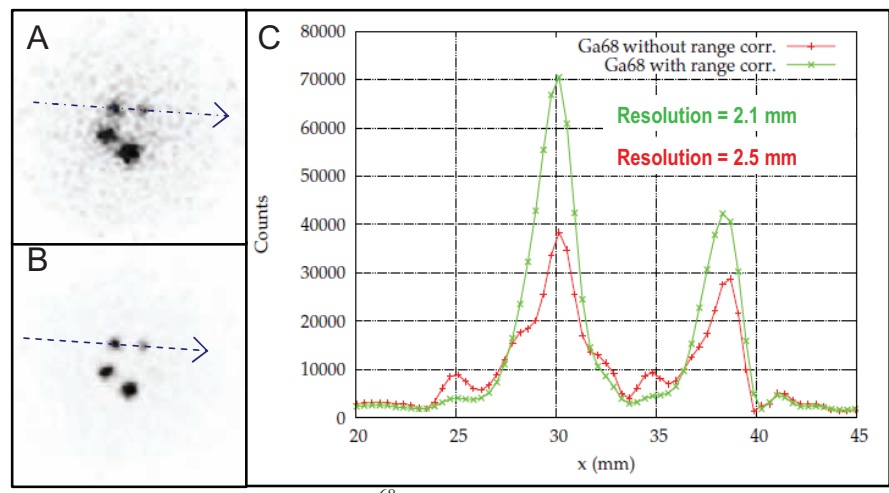

Fig. 2. IQ phantom filled with ${ }^{68} \mathrm{Ga}$ reconstructed with 3D OSEM. Without range corrections (A) and with range corrections (B). 10\% noise images are compared. In panel $\mathrm{C}$, count line profiles along the 2 and $3 \mathrm{~mm}$ rods (arrow in the images) are shown.

TABLE II: RESOLUTION AND RECOVERY COEFICIENT VALUES FOR A GIVEN NOISE LEVEL, OBTAINED FOR THE 3D OSEM RECONSTRUCTION WITH AND WITHOUT RANGE CORRECTION OF AN IQ PHANTOM FILLED WITH ${ }^{68} \mathrm{Ga}$,

\begin{tabular}{ccccc} 
& \multicolumn{2}{c}{$\begin{array}{c}{ }^{68} \mathrm{Ga} \text { (without } \\
\text { range correction) }\end{array}$} & \multicolumn{2}{c}{$\begin{array}{c}{ }^{68} \mathrm{Ga} \text { (with range } \\
\text { correction) }\end{array}$} \\
\hline $\begin{array}{c}\text { Noise } \\
(\%)\end{array}$ & $\begin{array}{c}\text { Resol. } \\
(\mathrm{mm})\end{array}$ & $\begin{array}{c}\mathrm{RC} \\
(\%)\end{array}$ & $\begin{array}{c}\text { Resol. } \\
(\mathrm{mm})\end{array}$ & $\begin{array}{c}\mathrm{RC} \\
(\%)\end{array}$ \\
\hline 6.0 & 2.68 & 39.3 & 2.43 & 47.7 \\
8.0 & 2.57 & 40.4 & 2.23 & 57.7 \\
10.0 & 2.53 & 40.5 & 2.06 & 67.8 \\
12.0 & 2.50 & 40.4 & 1.98 & 74.0 \\
14.0 & 2.48 & 40.5 & 1.93 & 78.2
\end{tabular}

\section{Image reconstruction using different materials.}

Fig. 3 shows the images reconstructed of an IQ phantom filled with ${ }^{18} \mathrm{~F}$ and ${ }^{68} \mathrm{Ga}$, employed to simulate positron range during reconstruction a density map of the different materials employed in this simulation. In the first case, without range correction, the reconstructed size of the ${ }^{18} \mathrm{~F}$ and ${ }^{68} \mathrm{Ga}$ line sources in water is larger than in bone, which causes an important difference in the respective heights of the activity profiles in bone and water. When positron range is modeled into the reconstruction, similar apparent size for the line source, and identical height of the activity profiles, are obtained for annihilations in the two materials.

The bone and water attenuation effects have not been taken into account in this work.

Table III shows the full width at half maximum (FWHM) of the ${ }^{18} \mathrm{~F}$ and ${ }^{68} \mathrm{Ga}$ line sources, in bone and water, after 3D OSEM reconstruction with and without positron range corrections. Most of the positron range effects have been removed, making the FWHM obtained for ${ }^{68} \mathrm{Ga}$ and ${ }^{18} \mathrm{~F}$ much more similar.

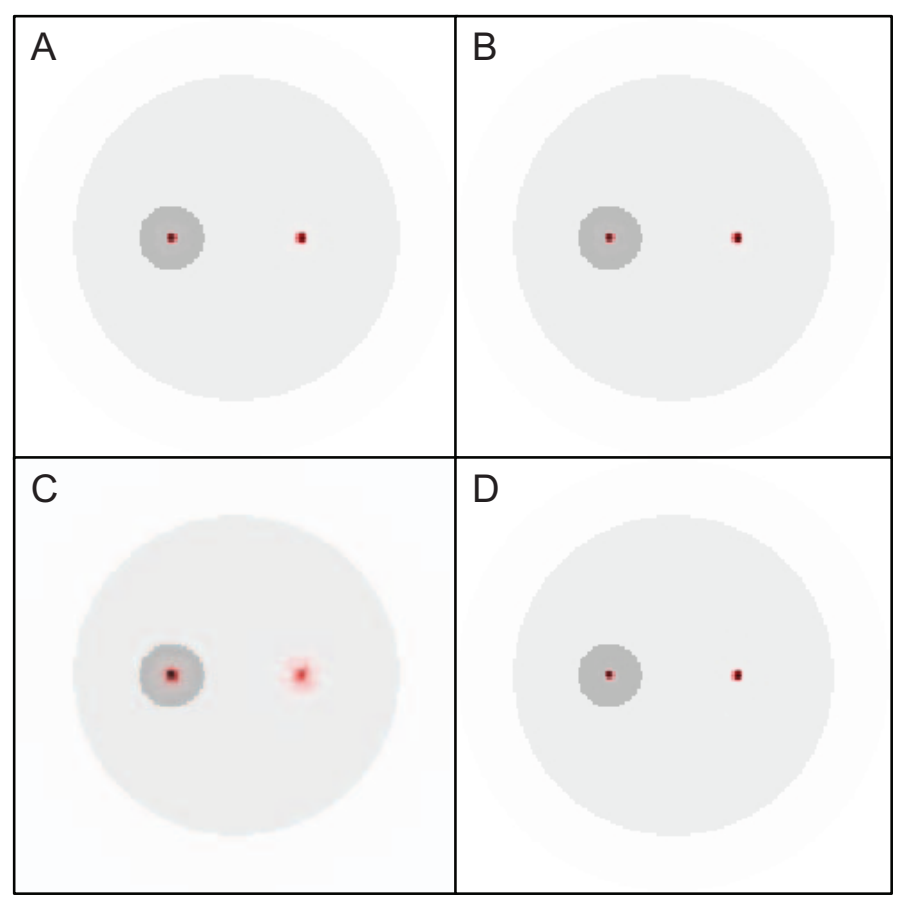

Fig. 3. Density map of simulated phantom superimposed to the reconstructed images of two ${ }^{18} \mathrm{~F}$ (panels $\mathrm{A}$ and $\mathrm{B}$ ) and two ${ }^{68} \mathrm{Ga}$ (panels $\mathrm{C}$ and D) line sources in bone and water after 3D OSEM reconstruction. Without range corrections $(\mathrm{A}, \mathrm{C})$ and with range corrections $(\mathrm{B}, \mathrm{D})$. Images are compared at $10 \%$ noise.

\begin{tabular}{|c|c|c|c|c|}
\hline \multirow[t]{2}{*}{ FWHM (mm) } & \multicolumn{2}{|c|}{$\begin{array}{l}\text { Without range } \\
\text { correction }\end{array}$} & \multicolumn{2}{|c|}{$\begin{array}{l}\text { With range } \\
\text { correction }\end{array}$} \\
\hline & Bone & Water & Bone & Water \\
\hline${ }^{18} \mathrm{~F}$ & 1.09 & 1.13 & 1.03 & 1.02 \\
\hline${ }^{68} \mathrm{Ga}$ & 1.62 & 2.01 & 1.22 & 1.23 \\
\hline
\end{tabular}

\section{CONCLUSION}

Positron range limits the spatial resolution of PET images. We model positron range effects in the 3D-OSEM reconstruction algorithm by means of a blurring kernel based on the material-dependent radial profile of the annihilation points obtained with PeneloPET simulations. This approach has a small computational cost, while it improves significantly the quality of the reconstructed images, rendering material dependent range corrected images for large positron range isotopes, like ${ }^{68} \mathrm{Ga}$, practical and useful.

\section{ACKNOWLEDGMENT}

This work has been supported in part by MEC (FPA200762216), CDTEAM (Programa CENIT, Ministerio de Industria), UCM (Grupos UCM, 910059), CPAN (Consolider-Ingenio 2010) CSPD-2007-00042 and the RECAVA-RETIC network. Part of the calculations of this work were performed in the "Clúster de Cálculo de Alta Capacidad para Técnicas Físicas" funded in part by UCM and in part by UE under FEDER programme". 


\section{REFERENCES}

[1] C.S. Levin and E.J. Hoffman, Calculation of Positron Range and Its Effect on the Fundamental Limit of Positron Emision Tomography System Spatial Resolution, Phys. Med. Biol., 44:781 99, 1999.

[2] M.E. Phelps, E.J. Hoffman and S. Huang, Effects of Positron Range on Spatial Resolution, J. Nucl. Med., 16:649 52, 1975.

[3] W. Yuchuan, J. Seidel, B.M.W. Tsui, J.J Vaquero, M.G. Pomper, "Performance Evaluation of the GE Helthcare eXplore VISTA Dual Ring Small Animall PET Scanner", J. Nucl. Med. Vol. 47 No.11 1891 1900 .

[4] S.E. Derenzo, Mathematical Removal of Positron Range Blurring in High Resolution Tomography, IEEE Trans. Nucl. Sci., 33(1):565 9, 1986.

[5] B. Bai, A. Ruangma, R. Laforest, Y.C. Tai and R.M. Leahy. Positron range modeling for statistical PET image reconstruction. IEEE Nucl. Sci. Symposium Conference Record, pp 2501 2505, 2003.

[6] B. Bai, R. Laforest, A.M. Smith and R.M. Leahy, Evaluation of MAP image reconstruction with positron range modeling for 3D PET, IEEE Nucl. Sci. Symposium Conference Record, pp 2686 2689, 2005.

[7] Lin Fu, Jinyi Qi, A novel iterative image reconstruction method for high resolution imaging with a Monte Carlo based Positron Range model, IEEE Nucl. Sci. Symposium Conference Record, pp , 2008.

[8] J. L. Herraiz, S. España, J. J. Vaquero, M. Desco, and J. M. Udias, FIRST: Fast Iterative Reconstruction Software for (PET) tomography, IEEE Trans. Nucl. Sci., 2003, vol. 50, pp. 398404.

[9] Z.H. Cho, J.K. Chan, L. Ericksson, M. Singh, S. Graham, N.S. Macdonald and Y. Yano, Positron Ranges Obtained From Biomedically Important Positron Emitting Radionuclides, J. Nucl. Med., 16:1174 6, 1975.

[10] S.E. Derenzo, Precision Measurement of Annihilation Point Spread Distributions for Medically Important Positron Emitters, Positron Annihilation R.R Hasiguti and K. Fujiwara, Eds. Sendai, Japan: The Japan Institute of Metals, 819 23, 1979.

[11] C. Champion and C. L. Loirec, Positron follow up in liquid water: II. spatial and energetic study for the most important radioisotopes used in PET. Phys. Med. Bio., vol. 52, no. 22, pp. 6605 6625, 2007.

[12] A. Alessio and L. MacDonald, Spatially Variant Positron Range Modeling Derived from CT for PET Image Reconstruction. IEEE Nucl. Sci. Symposium Conference Record, pp 2912 2915, 2009.

[13] S. España, J.L. Herraiz, E. Vicente, J.J. Vaquero, M. Desco, J.M. Udias, PeneloPET, a Monte Carlo PET simulation tool based on PENELOPE: features and validation, Phy Med Biol, 54, 172342.

[14] NEMA N 2008 Performance Measurements for Small Animal Positron Emission Tomographs. (NEMA: National Electrical Manufacturers Association).

[15] L. Katz and A.S. Penfold, Rev. Mod. Phys., 24 (1952), p. 28.

[16] Kisung Lee et al., "Impact of system design parameters on image figures of merit for a mouse PET scanner" IEEE TSN, vol. 51, no. 1, feb. 2004.

[17] Evans RD: The Atomic Nucleus, New York, McGraw Hill, 1972, p 628. 